\title{
Small steps forward for HIV vaccine development
}

\author{
A trial of a therapeutic vaccine against HIV induces cellular immunity and, although it provides hope, it highlights \\ the hurdles for the development of such strategies.
}

\section{Beatriz Mothe and Christian Brander}

$\mathrm{n}$ people with established infection
by human immunodeficiency virus (HIV), host immunity to the virus has been linked to partial control of viral replication ${ }^{1-4}$. However, whether such immune responses are enough to mediate an antiretroviral treatment (ART)-free HIV remission state after interruption of ART or how to induce such immune responses by any intervention in HIV-infected people remain unsolved challenges. In this issue of Nature Medicine, Colby et al. report a preliminary study of a therapeutic vaccine in HIV-infected people who had initiated ART early after infection ${ }^{5}$.

In the vast majority of HIV-infected people on ART, after discontinuation of ART (ART interruption), replication-competent proviruses in the viral reservoir drive a rapid viral rebound within 2-4 weeks ${ }^{6}$. Interestingly, in several studies, lower levels of viral reservoir have been associated with a delayed time to viral rebound ${ }^{7}$. Even though complete eradication may not be achieved, many current cure strategies aim to mobilize and reduce the viral reservoir to levels that would allow an effective, sustained control of the virus.

It is well known that people able to initiate ART within the first weeks after HIV acquisition typically show lower viral reservoir levels, less exhaustion of the immune system ${ }^{8}$ and fewer immuneadapted viral variants than those of people who initiate ART at later stages of infection ${ }^{9}$. Thus, people treated early with ART have been hypothesized to be able to induce a protective immune response able to control their viral reservoir after therapeutic vaccination, since the reservoir is smaller and less adapted. This is also supported by the notion that up to $10-15 \%$ of people treated early may be able to maintain viremia at undetectable or low levels after cessation of ART (post-treatment controllers) without the need for any immune intervention ${ }^{10,11}$. While the precise mechanisms that lead to post-treatment control are still elusive and may differ from the mechanisms involved in spontaneous control of HIV, it seems plausible that host

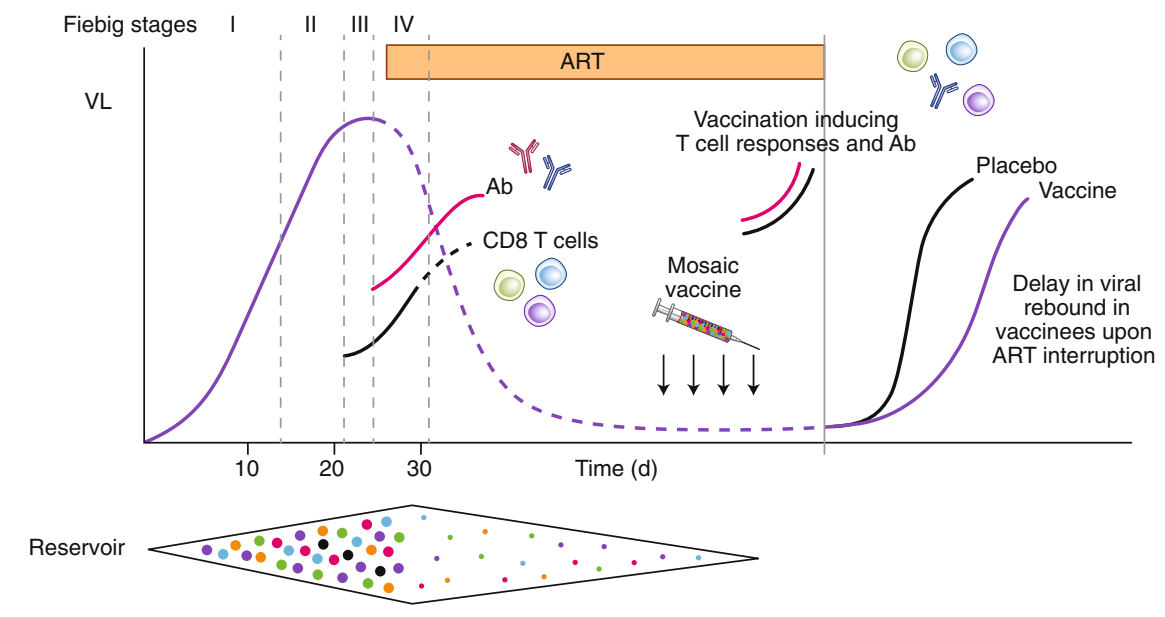

Fig. 1 | Schematic representation of HIV viral load (VL) kinetics during acute and recent HIV infection and upon antiretroviral treatment interruption. Viral reservoir seeding occurs after the first days of HIV acquisition, and cellular and humoral responses are induced during acute and early HIV infection while the reservoir increases progressively in viral diversity. Mosaic vaccines are designed to tackle HIV diversity and to induce both antibody and HIV-specific T cells. In the study by Colby et al., mosaic vaccines were immunogenic and able to delay HIV viral rebound after antiretroviral treatment interruption. Ongoing studies will clarify how vaccine-induced responses match the sequences of preexisting and rebounding viruses.

immune response induced during primary infection and preserved by early ART may have a critical role in the capacity of these people to contain viral rebound ${ }^{12}$.

Colby et al. carried out a study of a therapeutic vaccine in 26 ART-suppressed HIV-infected people in Thailand who were treated with ART within the earliest stages of their infection with HIV (Fiebig stages I-IV) 5 . Vaccination in this study (RV405) included a four-shot vaccination regimen using vaccines with two different viral vectors, Ad26 (adenovirus) and MVA (modified vaccinia Ankara), that express two multivalent mosaic immunogens from the HIV proteins Gag, Pol and Env; the vaccines were given twice each over a period of 12 months. These novel mosaic immunogens were designed in silico to increase the vaccine coverage of potential $\mathrm{T}$ cell epitopes in circulating viruses and aimed to tackle the diversity of HIV type 1 and limit the virus's ability to escape immunosurveillance. In previous experiments in a non-human primate model of simian immunodeficiency virus, mosaic vaccines, given alone or in combination with an immune modulator TLR7 agonist, showed both a delay in viral rebound and a significant reduction in the viral setpoint after interruption of ART, in animals treated early ${ }^{13,14}$. Notably, in the non-human primate study, time to viral rebound and viral setpoint correlated with levels of vaccine-induced $\mathrm{T}$ cells, which supports the clinical testing of this strategy.

Colby et al. found that in humans, the intervention was safe and immunogenic and induced high levels of polyfunctional $\mathrm{CD}^{+} \mathrm{T}$ cells and $\mathrm{CD} 8^{+} \mathrm{T}$ cells and Env-specific antibodies ${ }^{5}$. During an exploratory ART-interruption phase, the 17 participants in the active arm showed a statistically non-significant delay in the time to viral rebound from 21 days, compared with the 15 days to viral rebound for those on placebo controls (Fig. 1). All people, except one placebo recipient, had to resume treatment as per the protocol, since 
they met the criteria for two consecutive determinations of viremia $>1,000$ copies per $\mathrm{ml}, 1$ week apart. As the authors concluded, the trend in delayed rebound was too short to positively affect clinical management of the infection, but it partially reproduced the delayed rebound observed in the nonhuman primate model.

Still, despite the modest clinical effect, we feel that a number of lessons can be learned from the study by Colby et al., especially with regard to future trial design and possible readouts in the cure field. First, the question of whether a placebo group is indeed needed for scientific validity in pilot studies involving such unique and scarce HIV groups that also present an almost-universal viral rebound ${ }^{15}$ and for which efficacy comparisons among groups cannot be sufficiently powered. Second, stratification by HLA genotype upon randomization between arms might be necessary to limit the potential effects of host genetics on the results, as highlighted in the present RV405 study. As alluded to by the authors, the conservative ART-resumption criteria used in the study here may have blunted the possibility of detecting any potential post-rebound control of the virus after an initial phase of transient viremia. Such post-rebound control may occur only after vaccine-induced memory $\mathrm{T}$ cell responses are first re-stimulated by the rebounding virus to regain full effector functions and/or home to the relevant tissue to eliminate infected cells. Alternatively, innate immune mechanisms known as 'vaccinial effects' ${ }^{\prime 6}$ triggered by a transient viremia may also be required before the virus can be effectively controlled. Since more than $30 \%$ of cases of post-treatment control can occur after transient viremia higher than 10,000 copies per milliliter (ref. ${ }^{11}$ ), more-relaxed ART-resumption criteria may need to be employed to offer those entering an analytic treatment interruption (ATI) the possibility of achieving an effective post-rebound control ${ }^{16}$. However, elevated levels of viremia over a prolonged period of time also considerably increase the risk for viral transmission during ATI, and any such approach would require effective risk mitigation to avoid such transmission.

As show in the study by Colby et al., therapeutic vaccination was able to induce robust and broad immune responses ${ }^{5}$. However, it remains to be determined whether the lack of control of the virus may have been due to still-insufficient stimulation of immune responses, inadequate response profiles, lack of reservoir mobilization, limited coverage of autologous viruses or the expansion of $\mathrm{T}$ cell and $\mathrm{B}$ cell responses to irrelevant targets in the virus. Comparison analyses of sequences of pre-existing and rebounding viruses, known as 'sieve effect' analyses, as well as detailed characterization of host immunity before ART initiation and during ATI, will hopefully help to elucidate the mechanisms that determine the rebound kinetics.

Finally, the results from Colby et al. ${ }^{5}$ cannot hide the fact that the HIV cure field is still a long way from reproducing the recent advances made in the non-human primate model. In particular, there is an urgent need to better understand mechanisms associated with primary infection and viral rebound during ATI, not only to facilitate the design of new interventions aimed at reproducing post-treatment controller phenotypes but also to help to optimize cure trial designs. However, the positive indications from the work from Colby et al. ${ }^{5}$ clearly warrant further testing of improved vaccines and/or combinational strategies.

\section{Beatriz Mothe (1D) 1,2,3凶 and \\ Christian Brander 2,3,4,5 \\ ${ }^{1}$ Infectious Diseases Department, Hospital \\ Universitari Germans Trias i Pujol, Badalona, Spain. ${ }^{2}$ IrsiCaixa AIDS Research Institute, Badalona, Spain. ${ }^{3}$ Universitat de Vic-Central of Catalonia, Vic, Spain. ${ }^{4}$ AELIX Therapeutics, Barcelona, Spain. ${ }^{5} I C R E A$, Barcelona, Spain.

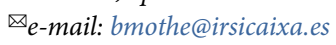

Published online: 2 April 2020

https://doi.org/10.1038/s41591-020-0837-0

\section{References}

1. Koup, R. A. et al. J. Virol. 68, 4650-4655 (1994).

2. Cartwright, E. K. et al. Immunity 45, 656-668 (2016).

3. Betts, M. R. et al. Blood 107, 4781-4789 (2006).

4. O’Brien, S. J., Gao, X. \& Carrington, M. Trends Mol. Med. 7, 379-381 (2001).

5. Colby, D.J. et al. Nat. Med. https://doi.org/10.1038/s41591-0200774-y (2020).

6. El-Sadr, W. M. et al. N. Engl. J. Med. 355, 2283-2296 (2006)

7. Li, J. Z. et al. AIDS 30, 343-353 (2016).

8. Robb, M. L. \& Ananworanich, J. Curr. Opin. HIV AIDS 11, 555-560 (2016).

9. Deng, K. et al. Nature 517, 381-385 (2015).

10. Sáez-Cirión, A. et al. PLoS Pathog. 9, e1003211 (2013).

11. Namazi, G. et al. J. Infect. Dis. 218, 1954-1963 (2018).

12. Streeck, H. et al. J. Virol. 83, 7641-7648 (2009).

13. Borducchi, E. N. et al. Nature 540, 284-287 (2016).

14. Nishimura, Y. et al. Nature 543, 559-563 (2017).

15. Muccini, C. et al. AIDS Res. Ther. 16, 25 (2019)

16. Julg, B. et al. Lancet HIV 6, e259-e268 (2019).

\title{
Unraveling the genetic contributions to complex traits across different ethnic groups
}

\author{
Trans-ethnic study shows promise in the identification of genetic commonalities and differences for the \\ contribution of traits to lifespan across genetically diverse populations.
}

Karen A. Mather and Anbupalam Thalamuthu

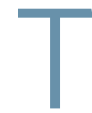
he use of diverse ethnic and racial groups in genetic studies is vital for identification of the genetic risk factors unique to specific populations and for determining whether the results can be generalized to other populations. However, a major shortcoming of human geneticassociation studies has been the relative lack of non-European participants, despite more than $75 \%$ of the world's population being of African or Asian ancestry ${ }^{1}$. For example, as of early 2019 , only about $22 \%$ of participants in the commonly used genomewide association study (GWAS) paradigm were non-European ${ }^{2}$. Furthermore, the 